\title{
Coronary artery disease and myocardial revascularization in patients undergoing transcatheter aortic valve replacement
}

\author{
Raffaele Piccolo, Anna Franzone, Thomas Pilgrim \\ Department of Cardiology, Swiss Cardiovascular Center, Bern University Hospital, University of Bern, Bern, Switzerland \\ Correspondence to: Prof. Thomas Pilgrim, MD. Department of Cardiology, Swiss Cardiovascular Center, Bern University Hospital, University of \\ Bern, CH-3010 Bern, Switzerland. Email: thomas.pilgrim@insel.ch. \\ Provenance: This is an invited Editorial commissioned by Section Editor Dr. Hui-Ping Zhang (Department of Cardiology, Beijing Hospital, the Fifth \\ Affiliated Hospital of Peking University, Beijing, China). \\ Comment on: Kotronias RA, Kwok CS, George S, et al. Transcatheter Aortic Valve Implantation With or Without Percutaneous Coronary Artery \\ Revascularization Strategy: A Systematic Review and Meta-Analysis. J Am Heart Assoc 2017;6. pii: e005960.
}

Submitted Sep 19, 2017. Accepted for publication Oct 01, 2017.

doi: $10.21037 /$ jtd.2017.10.20

View this article at: http://dx.doi.org/10.21037/jtd.2017.10.20

Aortic stenosis (AS) is frequently interwoven with coronary artery disease (CAD) due to shared risk factors. As a consequence, $\mathrm{CAD}$ is found in a substantial proportion of patients undergoing transcatheter aortic valve replacement (TAVR) in contemporary studies, ranging between $45 \%$ and $75 \%$ according to various definitions. Inevitably, the coexistence of the two conditions poses additional questions to their respective management and clinicians are typically confronted with at least three issues: (I) what is the prognostic relevance of concomitant CAD in TAVR patients? (II) what is the short- and long-term outcome of percutaneous coronary intervention (PCI) in TAVR candidates? (III) what is the optimal timing for PCI in relation to the TAVR procedure: before, during or after?

Against this background of lingering questions, Kotronias and colleagues performed a systematic review and meta-analysis of observational studies in order to explore the benefit of PCI among patients undergoing TAVR (1). We structured the appraisal of their work according to the three questions raised above.

\section{Clinical implications of obstructive CAD in TAVR candidates}

In a study of age- and gender-matched cohorts, concomitant CAD in TAVR patients has been associated with a $75 \%$ relative risk increase in the composite of cardiovascular death, myocardial infarction, or stroke at 1 year (2).
Interestingly, the risk of ischemic events during 1-year follow-up among TAVR patients without CAD was similar compared to that of a matched population of CAD patients undergoing PCI during 1-year follow-up (2). Along the same line, in a TAVR registry from the United Kingdom, the presence of CAD was significantly associated with increased mortality at 2 years of follow-up (3). Taken together, these data suggest that the presence of CAD affects longterm prognosis, while its absence confers more favorable outcomes. It is important to note that the results remain conflicting in view of other studies negating a prognostic role of obstructive CAD by applying similar definitions (4). However, data become more concordant in supporting a role of CAD particularly in the subset of patients with intermediate-to-high SYNTAX scores $(4,5)$.

\section{Safety and effectiveness of PCI in patients with CAD undergoing TAVR}

Conceptually, it is tempting to speculate that myocardial revascularization by means of PCI may reverse the detrimental impact of obstructive CAD on clinical outcomes. To address this issue, Kotronias and colleagues performed a meta-analysis by pooling data from nine observational studies involving 3,858 patients undergoing TAVR (1). As main results, the authors found that patients who underwent PCI experienced a higher risk of mortality at 30 days [odds ratio (OR), 1.42; 95\% confidence intervals 
(CI), 1.08-1.87] but not at 1 year (OR, 1.05; 95\% CI, $0.71-$ 1.56). Furthermore, the increased risk of death during early follow-up was not attributable to cardiovascular mortality (OR, 1.03; 95\% CI, 0.35-2.99). With the exception of major vascular complications (OR, 1.86; 95\% CI, 1.33-2.60), there was no difference between TAVR in combination with PCI $v s$. TAVR alone in terms of myocardial infarction, stroke, major or life-threatening bleeding, and acute kidney injury. When data from this meta-analysis are interpreted, we need to carefully consider the following aspects. First, data was derived from observational studies in which the decision to perform PCI or not was not randomly allocated. As such, findings are heavily affected by selection bias and confounding factors. Second, the authors pooled crude numbers of events and not maximally adjusted risk estimates that, in case of availability, would have attenuated but not cancelled out the observational nature of pooled studies. Third, as also acknowledged in the article, the increased risk of mortality observed at 30 days in the PCI group was mainly driven by a single study, which weighted $46 \%$ in their analysis. Contrariwise, after the exclusion of this study risk estimates showed a neutral effect of PCI for 30 days mortality as well as no difference in terms of mortality at longer term follow-up.

\section{Timing of $\mathrm{PCI}$ in relation to TAVR}

Another interesting analysis undertaken by Kotronias and colleagues explored the effect of timing of PCI in patients undergoing TAVR, even though only two studies contributed to the analysis (1). There was no significant difference in terms of mortality, myocardial infarction, stroke and other complications between patients undergoing PCI before TAVR and those who underwent concomitant procedures, i.e., PCI and TAVR during the same session.

As there is no clear advantage between the two strategies, these results support the concept that optimal timing of PCI should be customized in each patient. For example, in patients with chronic kidney dysfunction or in those with complex anatomies requiring larger amount of contrast, a staged procedure should be performed in order to limit the risk of contrast-induced acute kidney injury. Based also on the results of this meta-analysis, showing that patients undergoing PCI may have a higher risk of major vascular complications, radial vascular access may be preferred to the femoral route given its strong effect in decreasing the risk of vascular complications and major bleeding (6). Conversely, there are some specific cases in which a combined procedure may be favored. For example, in patients with simple lesions amenable to a straightforward stent implantation are ideal for a combined procedure. As there is an increased incidence of bleeding peaking in the aftermath of the TAVR procedure, PCI may be performed during TAVR in patients at higher risk of bleeding in order to obviate the use of dual antiplatelet therapy at the time of TAVR $(7,8)$. Furthermore, in patients with large area of myocardium at risk, such as in case of left main disease, a concomitant procedure may be preferentially used in view of a possible rapid deterioration of myocardial function and hemodynamic compromise associated with transient blood flow interruption during PCI in the left main coronary artery. Finally, concomitant procedures may reduce the need for unplanned rehospitalizations, which are associated in turn with an increased risk of mortality (9). Irrespective of timing, new-generation drug-eluting stents should be preferred to early-generation drug-eluting stents and bare metal stents in all patients requiring PCI based on established evidence proving both safety and efficacy $(10,11)$.

It is important to underscore that available data has primarily been derived from high-risk or inoperable patients undergoing TAVR. As the incidence of CAD is positively associated with ageing, results may change by extending TAVR to lower risk patients or other specific conditions such as pure aortic regurgitation $(12,13)$.

\section{Conclusions}

Although CAD is frequently observed among patients undergoing TAVR, its prognostic role and treatment strategy remain still controversial in view of the observational nature of evidence summarized now by Kotronias and colleagues (1). Several randomized trials designed to investigate revascularization modalities and the optimal timing of intervention in patients undergoing TAVR are ongoing (ISRCTN75836930, NCT01586910, NCT02797158) and their results will guide decisionmaking in clinical practice.

\section{Acknowledgements}

None.

\section{Footnote}

Conflicts of Interest: T Pilgrim received research grants to the institution from Symetis, Biotronik, and Edwards 
Lifesciences. The other authors have no conflicts of interest to declare.

\section{References}

1. Kotronias RA, Kwok CS, George S, et al. Transcatheter Aortic Valve Implantation With or Without Percutaneous Coronary Artery Revascularization Strategy: A Systematic Review and Meta-Analysis. J Am Heart Assoc 2017;6. pii: e005960.

2. Franzone A, Stortecky S, Raber L, et al. Effects of coronary artery disease in patients undergoing transcatheter aortic valve implantation: A study of age- and gender-matched cohorts. Int J Cardiol 2017;243:150-5.

3. Ludman PF, Moat N, de Belder MA, et al. Transcatheter aortic valve implantation in the United Kingdom: temporal trends, predictors of outcome, and 6-year followup: a report from the UK Transcatheter Aortic Valve Implantation (TAVI) Registry, 2007 to 2012. Circulation 2015;131:1181-90.

4. Witberg G, Regev E, Chen S, et al. The Prognostic Effects of Coronary Disease Severity and Completeness of Revascularization on Mortality in Patients Undergoing Transcatheter Aortic Valve Replacement. JACC Cardiovasc Interv 2017;10:1428-35.

5. Stefanini GG, Stortecky S, Cao D, et al. Coronary artery disease severity and aortic stenosis: clinical outcomes according to SYNTAX score in patients undergoing transcatheter aortic valve implantation. Eur Heart J 2014;35:2530-40.

6. Piccolo R, Galasso G, Capuano E, et al. Transradial versus transfemoral approach in patients undergoing percutaneous coronary intervention for acute coronary

Cite this article as: Piccolo R, Franzone A, Pilgrim T. Coronary artery disease and myocardial revascularization in patients undergoing transcatheter aortic valve replacement. J Thorac Dis 2017;9(11):4219-4221. doi: $10.21037 /$ jtd.2017.10.20 syndrome. A meta-analysis and trial sequential analysis of randomized controlled trials. PLoS One 2014;9:e96127.

7. Piccolo R, Pilgrim T, Franzone A, et al. Frequency, Timing, and Impact of Access-Site and Non-AccessSite Bleeding on Mortality Among Patients Undergoing Transcatheter Aortic Valve Replacement. JACC Cardiovasc Interv 2017;10:1436-46.

8. Stortecky S, Stefanini GG, Pilgrim T, et al. Validation of the Valve Academic Research Consortium Bleeding Definition in Patients With Severe Aortic Stenosis Undergoing Transcatheter Aortic Valve Implantation. J Am Heart Assoc 2015;4:e002135.

9. Franzone A, Pilgrim T, Arnold N, et al. Rates and predictors of hospital readmission after transcatheter aortic valve implantation. Eur Heart J 2017;38:2211-7.

10. Piscione F, Piccolo R, Cassese S, et al. Clinical impact of sirolimus-eluting stent in ST-segment elevation myocardial infarction: a meta-analysis of randomized clinical trials. Catheter Cardiovasc Interv 2009;74:323-32.

11. Piccolo R, Pilgrim T, Heg D, et al. Comparative Effectiveness and Safety of New-Generation Versus Early-Generation Drug-Eluting Stents According to Complexity of Coronary Artery Disease: A Patient-Level Pooled Analysis of 6,081 Patients. JACC Cardiovasc Interv 2015;8:1657-66.

12. Franzone A, Pilgrim T, Stortecky S, et al. Evolving Indications for Transcatheter Aortic Valve Interventions. Curr Cardiol Rep 2017;19:107.

13. Franzone A, Piccolo R, Siontis GC, et al. Transcatheter Aortic Valve Replacement for the Treatment of Pure Native Aortic Valve Regurgitation: A Systematic Review. JACC Cardiovasc Interv 2016;9:2308-17. 